\title{
Long Non-Coding RNAs in Vascular Inflammation
}

\author{
Stefan Haemmig, Viorel Simion and Mark W. Feinberg* \\ Cardiovascular Division, Department of Medicine, Brigham and Women's Hospital, Harvard Medical School, Boston, MA, \\ United States
}

Less than $2 \%$ of the genome encodes for proteins. Accumulating studies have revealed a diverse set of RNAs derived from the non-coding genome. Among them, long noncoding RNAs (IncRNAs) have garnered widespread attention over recent years as emerging regulators of diverse biological processes including in cardiovascular disease (CVD). However, our knowledge of their mechanisms by which they control CVD-related gene expression and cell signaling pathways is still limited. Furthermore, only a handful of IncRNAs has been functionally evaluated in the context of vascular inflammation, an important process that underlies both acute and chronic disease states. Because some IncRNAs may be expressed in cell- and tissue-specific expression patterns, these non-coding RNAs hold great promise as novel biomarkers and as therapeutic targets in health and disease. Herein, we review those IncRNAs implicated in pro- and anti-inflammatory processes of acute and chronic vascular inflammation. An improved understanding of IncRNAs in vascular inflammation may provide new pathophysiological insights in CVD and opportunities for the generation of a new class of RNA-based biomarkers and therapeutic targets.

Yuichi Oike,

Kumamoto University, Japan Anna Zampetaki,

King's College London School of Medicine, United Kingdom

*Correspondence:

Mark W. Feinberg mfeinberg@partners.org

Specialty section: This article was submitted to

Atherosclerosis and

Vascular Medicine,

a section of the journal

Frontiers in Cardiovascular Medicine

Received: 18 November 2017 Accepted: 28 February 2018

Published: 14 March 2018

Citation:

Haemmig S, Simion $V$ and Feinberg MW

(2018) Long Non-Coding RNAs in

Vascular Inflammation.

Front. Cardiovasc. Med. 5:22. doi: 10.3389/fcvm.2018.00022
Keywords: IncRNAs, vascular inflammation, cardiovascular disease, acute inflammation, chronic inflammation

\section{INTRODUCTION}

Accumulating studies highlight that inflammatory processes and traditional cardiac risk factors may cooperatively contribute to vascular disease leading to the development of cardiovascular events (1). A variety of systemic inflammatory diseases such as rheumatoid arthritis (RA), systemic lupus erythematosus, psoriatic arthritis, and medium to large vessel vasculitis are associated with an increased risk of atherosclerotic events and premature cardiovascular disease (CVD) (2). Interestingly, acute inflammation (e.g., sepsis) also significantly increases the risk of future cardiovascular events (3). Although these diseases differ in their autoimmune and/or inflammatory nature, atherosclerosis may represent a common response with local vascular inflammation in subintimal and perivascular layers. Over decades, a progressive inflammatory multistep process in lesion-prone regions of the arterial vasculature develops by different disease-specific upstream insults $(1,4)$. However, our understanding of the pathophysiological links between systemic inflammatory diseases to vascular inflammation remains poorly understood. The recent recognition that as much as $70-90 \%$ of the genome is pervasively transcribed at some point during development has opened new opportunities to address these questions (5-7). Most of those transcripts are non-coding measuring greater than 200 nucleotides in length and display mRNA-like processing properties. This class of non-coding RNA (ncRNA) is known as long ncRNAs (lncRNAs). Whereas the number of $\sim 19,000$ human protein-coding genes has plateaued, the number of lncRNAs keeps increasing annually. However, it should be pointed out that lncRNAs have a low cross-species conservation rate and many lncRNAs have extremely low expression 
levels per cell (8-10). Nevertheless, lncRNAs have emerged as powerful regulators of nearly all biological processes by mediating epigenetic, transcriptional, or translational control of target genes due to their polyvalent binding properties to RNA, DNA, and protein as well as acting as molecular sponges for other transcripts and miRNAs (11). The subcellular localization pattern can provide additional insights into the mechanistic role for lncRNAs. Other considerations include whether the lncRNA acts in cis or trans and whether the RNA product itself is essential for fulfilling its function or if its transcription per se that underlies its function (12). However, the role of IncRNAs in vascular inflammation and CVD is just emerging (13). This review will summarize recent findings that provide mechanistic and translational insights of lncRNAs in acute and chronic vascular inflammation in the context of CVD.

\section{ACUTE INFLAMMATION}

The acute inflammatory response is induced as a first line of defense against microbial infection and other "non-self" stimuli. Antigenpresenting cells express different receptors, of which the Toll-like receptor (TLR) family is best characterized. TLRs are especially sensitive to microbes products such as LPS, lipoproteins, and nucleic acids (14). Once activated, these receptors trigger complex signaling cascades resulting in changes in expression of hundreds of genes involved in immunity and inflammation. TLRs have been implicated in destabilizing plaques leading to atherothrombosis in the vessel wall. For example, TLR4 enhances macrophage responses to lipids and inflammation, whereas TLR2 potentiates inflammation more broadly in the vessel wall in both macrophages and vascular cells, an effect that may lead to superficial erosion of atherosclerotic lesions $(15,16)$. Recent studies have connected a range of acute inflammatory processes to lncRNA expression and found that lncRNAs can regulate the acute inflammatory response, opening new avenues for exploring pathophysiological insights that may lead to improved disease stage-specific diagnostics and therapeutic interventions.

Metastasis-associated lung adenocarcinoma transcript 1 (MALAT1) is a conserved lncRNA whose expression correlates with many human cancers. Recent data also indicate its significance in immunity, and specifically in acute inflammation (17). MALAT1 expression is increased in LPS-activated macrophages (17), cardiac microvascular endothelial cells (CMVEC), and in the hearts of rats with sepsis (18). Knockdown of MALAT1 increases the LPS-induced expression of TNFa and IL-6 in macrophages. Mechanistically, MALAT1 interacts with NF- $\kappa \mathrm{B}$ in the nucleus to inhibit its DNA binding activity, and consequently decreases the production of inflammatory cytokines (17). MALAT1 was also found to interact with the polycomb protein EZH2 in CMVECs in response to LPS activation (18). In a recent study, MALAT1 expression was increased by LPS stimulation in murine cardiomyocytes and in cardiac tissue of a mouse sepsis model (19). MALAT1 overexpression enhanced TNFa production on LPS-stimulated cardiomyocytes, while MALAT1 siRNA had an inhibitory effect, via serum amyloid antigen 3 (SAA3), an inflammatory ligand that can stimulate IL- 6 and TNFa production, as observed in other cells such as endothelial (20) and mouse liver cells (21). Cardiomyocytes transfected with MALAT1 siRNA were less susceptible to LPSinduced cell apoptosis, suggesting that MALAT1 induction is a mechanism of cardiomyocyte apoptosis or injury in response to sepsis (19). Collectively, MALAT1 differentially regulates inflammatory responses in a cell-specific manner.

lincRNA-Cox2, is a lncRNA neighboring the Ptgs2 (Cox2) gene, recently discovered as a key regulator of inflammatory responses mediating both the activation and repression of distinct classes of immune genes (22). LPS stimulation induced lincRNA-Cox2 expression in both dendritic cells and bonemarrow derived macrophages (BMDM) in a similar pattern as Ptgs2 $(22,23)$. lincRNA-Cox2 expression is induced by LPS in a MyD88- and NF- $\mathrm{B}$-dependent manner and lincRNA-Cox2 silencing/overexpression in BMDM regulates important immune genes such as TNFa, IL-6, CCL5, SOCS3, and STAT3 (22). Several mechanisms have been described for lincRNA-Cox2, including interaction with heterogeneous nuclear ribonucleoprotein (hnRNP) A/B and A2/B1 (22), degradation of IKB- $\alpha$ in the cytosol, and assembly into the SWItch/Sucrose NonFermentable (SWI/SNF) complex, thereby acting as a co-activator of NF- $\kappa \mathrm{B}$ or inducing SWI/SNF-associated chromatin remodelling (24,

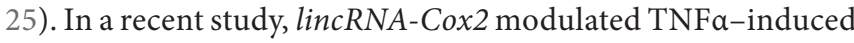
transcription of the IL-12b gene by promoting the recruitment of $\mathrm{Mi}-2 /$ nucleosome remodeling and deacetylase (Mi-2/NuRD) repressor complex to the IL-12b promoter region (26). Taken together, rapid activation of lincRNA-Cox 2 may regulate a range of acute inflammatory signaling pathways.

THRIL (TNFa and hnRNPL related immune-regulatory LincRNA) or linc1992, is a lncRNA that regulates TNFa expression through a negative feedback mechanism. THRIL binds specifically to heterogenous nuclear ribonucleoprotein $\mathrm{L}$ (hnRNPL) and forms a functional THRIL-hnRNPL complex that regulates transcription of the TNFa gene by binding to its promoter. THRIL is also required for expression of many immuneresponse genes and regulators of TNFa expression. Clinically, THRIL expression correlated with the severity of symptoms in patients with Kawasaki disease, an acute inflammatory disease of childhood (27). This study provides strong evidence that THRIL is required for induction of TNFa expression and plays an important role in acute inflammation and innate immunity. Further studies will be of interest to verify these findings in other inflammatory contexts, including chronic inflammation such as atherosclerosis or diabetes.

\section{CHRONIC INFLAMMATION}

Chronic inflammation is a major contributing factor to vascular events, including atherosclerotic plaque development, plaque erosion, aortic aneurysm, and ischemic myocardial damage. Inflammation disturbs the homeostasis of the endothelium, leading to endothelial dysfunction, which is amongst the earliest processes involved in atherosclerotic initiation (28). The early response is characterised by activation of endothelial cells (ECs), triggered by biochemical (e.g., IL-1 $\beta$, TNFa, oxLDL, etc.) and biomechanical stimulation in the form of disturbed blood flow [Rev by (29)]. Consequently, expression of adhesion 
molecules (e.g., VCAM-1, ICAM-1, E-Selectin) and secretion of membrane-associated chemokines (e.g., MCP-1, fractalkine) fosters the recruitment of monocytes and different types of $\mathrm{T}$ cells to the vessel wall (14-17). Chronic endothelial activation leading to the loss of endothelial integrity increases the risk for atherosclerosis. This is often observed in patients with RA, an autoimmune disease that causes chronic inflammation of the joints and systemically in the vasculature (30). A strong relationship exists between RA and atherosclerosis, but causality remains unclear.

Spurlock et al. identified that the expression of the lncRNA lincRNA-p21 is significantly lower specifically in patients with RA compared to healthy subjects. No dysregulation of lincRNA-p21 could be observed in systemic lupus erythematosus and Sjörgen's syndrome (31). Interestingly, lincRNA-p21 could be restored to normal levels in RA patients treated with methotrexate (MTX), which is the most commonly used anti-rheumatic drug with antiinflammatory properties (32). In vitro analysis of Jurkat T cells confirmed the induction of lincRNA-p21 by MTX (31). Initial work discovered lincRNA-p21 as a repressor of p53-dependent transcriptional responses. Silencing of lincRNA-p21 affected the expression of hundreds of genes known to be repressed by p53, which could be rescued by inhibiting p53, suggesting that lincRNA-p21 functions as a downstream repressor for $\mathrm{p} 53$. This transcriptional repression by lincRNA-p21 is mediated through an interaction with hnRNP-K (33). Because p53 expression levels positively correlated with lincRNA-p21 expression in RA patients, basal lincRNA-p21 expression may be p53-independent in PBMCs. In addition, $\operatorname{lin} c R N A-p 21$ was initially described to be regulated by $\mathrm{p} 53$ in the context of DNA damage response (33). To investigate whether specific inhibition of either ATM or DNAPKcs, two key upstream regulators of the DNA damage response $(34,35)$, could restore lincRNA-p21 or p53 expression, inhibition studies were performed. Indeed, MTX-mediated induction of p53 and lincRNA-p21 was blocked in Jurkat T cells treated with NU-7441 (i.e., inhibitor for DNA-PKcs), whereas there was no effect using low concentration of KU-55933 (inhibitor for ATM). Furthermore, using in vitro NF- $\mathrm{B}$ luciferase reporter assays, silencing of lincRNA-p21 abrogated MTX-mediated inhibition of NF- $\kappa \mathrm{B}$ activity. This effect could be simulated by using the NU-7441 inhibitor, demonstrating a link between lincRNA-p21 and DNA-PKcs-mediated regulation of NF- $\kappa B$ pathway (31). This finding is consistent with previous reports, demonstrating that DNA-PKcs is a regulator of inflammation by phosphorylating p50, a member of the NF- $\kappa$ B pathway (36). Collectively, these findings suggest that MTX decreases the NF- $\kappa \mathrm{B}$ pathway by increasing lincRNA-p21 levels through a DNA-PKcs-dependent mechanism (31).

Stuhlmüller et al. described high expression levels of the lncRNA H19 in synovial tissues and isolated synovial macrophages or synovial fibroblasts (SFB) from donor samples of RA patients compared to control subjects. H19 was also induced in SFB from RA ex vivo using multiple proinflammatory stimuli such as TNFa, IL-1 $\beta$, or PDGF-BB. Whether elevated levels of $\mathrm{H} 19$ in RA reflects its role as a biomarker of inflammatory stimuli or as a pathogenic mediator remains unknown $(37,38)$. Future studies will be required to further define the functional role of lncRNA H19 in RA pathogenesis and CVD.

Genome wide associated studies (GWAS) have identified the INK4b-ARF-INK4a locus located on chromosome 9p21 with multiple single nucleotide polymorphisms (SNPs) linked to coronary artery disease (CAD) (39-41), atherosclerosis (42), aortic aneurysm (43), ischemic stroke (41), type II diabetes (44) as well as specific cancer subtypes $(45,46)$. The lncRNA ANRIL (Antisense Non-coding RNA in the INK4 Locus) lies in opposite direction to the INK4b-ARF-INK4b locus, which contains the critical tumour suppressor genes $\mathrm{p} 14^{\mathrm{ARF}}, \mathrm{p} 15^{\mathrm{INK} 4 \mathrm{~b}}$ and $\mathrm{p} 16^{\mathrm{INK} 4 \mathrm{a}}(47)$. The SNPs associated with CAD do not correlate with well-established CAD risk factors, suggesting that this lncRNA is a novel independent driver for vascular inflammation. Specifically two ANRIL transcripts (EU741058 and NR_003529) are significantly increased from patients with $\mathrm{CAD}$ in human atherosclerotic plaque tissue as well as peripheral blood mononuclear cells, whereas the most abundant isoform DQ485454 is not differentially expressed (48). Loss-of-function studies reduced cell viability of SMCs for siRNAs targeting exclusively NR_003529 or both NR_003529 and DQ485454 isoforms (49). Moreover, ANRIL silencing increased the expression level of the antisense transcripts $\mathrm{p} 15^{\mathrm{ARF}}$ and $\mathrm{p} 16 \mathrm{INK} 4 \mathrm{~b}$, both key regulators for senescence, apoptosis, and stem cell selfrenewal by the retinoblastoma-p53 pathway abrogating PRC-1/2 binding to their loci (50). Additionally, ANRIL binds directly to PRC-1/2 components (i.e., CBX7 and/or SUZ12) supporting its role in regulating epigenetics (51). The multiple splice sites of ANRIL may result in isoform-specific effects, thus explaining some paradoxical findings. For example, an interesting isoform is circular ANRIL ( $c A N R I L)$, which results from exon skipping events during RNA splicing (52), adding another layer of complexity for the biological understanding of the ANRIL locus. cANRIL binds to PES1, an essential 60S-preribosomal assembly factor, impairing pre-rRNA processing and ribosome biogenesis in SMCs and macrophages. As a consequence, cANRIL induces nucleolar stress and p53 activation, resulting in the inhibition of proliferation and induction of apoptosis, as observed for the linear ANRIL (53). Although ANRIL is an independent risk factor for $\mathrm{CAD}$, its functional role in vascular inflammation in CVD still requires clarity based upon transcript specificity.

Hu et al. (54) identified increased expression of the lncRNA RP5-833A20.1 in human foam cells. Gain-of-function studies demonstrated that RP5-833A20.1 reduced cholesterol efflux and increased inflammatory cytokines, including IL- $1 \beta$, IL- 6 , and TNFa in THP-1 macrophages. Mechanistically, RP5-833A20.1 decreased the expression of NFIA by inducing miR-382-5 $\mathrm{p}$ expression. However, the specific mechanism of how RP5$833 A 20.1$ regulates miR-382-5 p expression for macrophage foam formation and verification of these findings in vivo will require further investigation (54).

Recently, Tontonoz et. al. demonstrated that in vivo delivery of the liver-expressed liver $\mathrm{X}$ receptor-induced lncRNA (LeXis) reduced aortic lesion size by Oil-red O staining (55). Since LeXis has been previously described to maintain hepatic sterol content and levels of serum cholesterol (56), the adenovirus- 
mediated LeXis overexpression in the liver was specifically designed using a thyroxine-binding globulin promoter. In line with their previous findings, LeXis overexpression reduced total serum cholesterol levels (55). This study raises the possibility for long-term lncRNA therapy in mice. Future studies that can overexpress $\operatorname{lncRNAs}$ in the liver or vessel wall may provide a novel therapeutic approach for regulating vascular inflammation in CVD.

Recent studies have illustrated increased HOTAIR expression in PBMCs and serum exosomes of RA patients, while lower expression of HOTAIR was detected in differentiated osteoclasts and rheumatoid synoviocytes. Overexpression of HOTAIR using lentivirus decreased the expression of IL-17, IL-23, IL-1 $\beta$, and TNF $\alpha$, and inhibited the activation of NF- $\mathrm{BB}$ in LPS-treated chondrocytes in a miR-138 regulated manner (57). These findings are in line with a previous exploratory study where HOTAIR expression was significantly reduced in LPS-treated chondrocytes and a RA mouse model (58). However, findings from acute inflammatory states such as sepsis are opposite. Using a mouse model of sepsis, HOTAIR expression was significantly

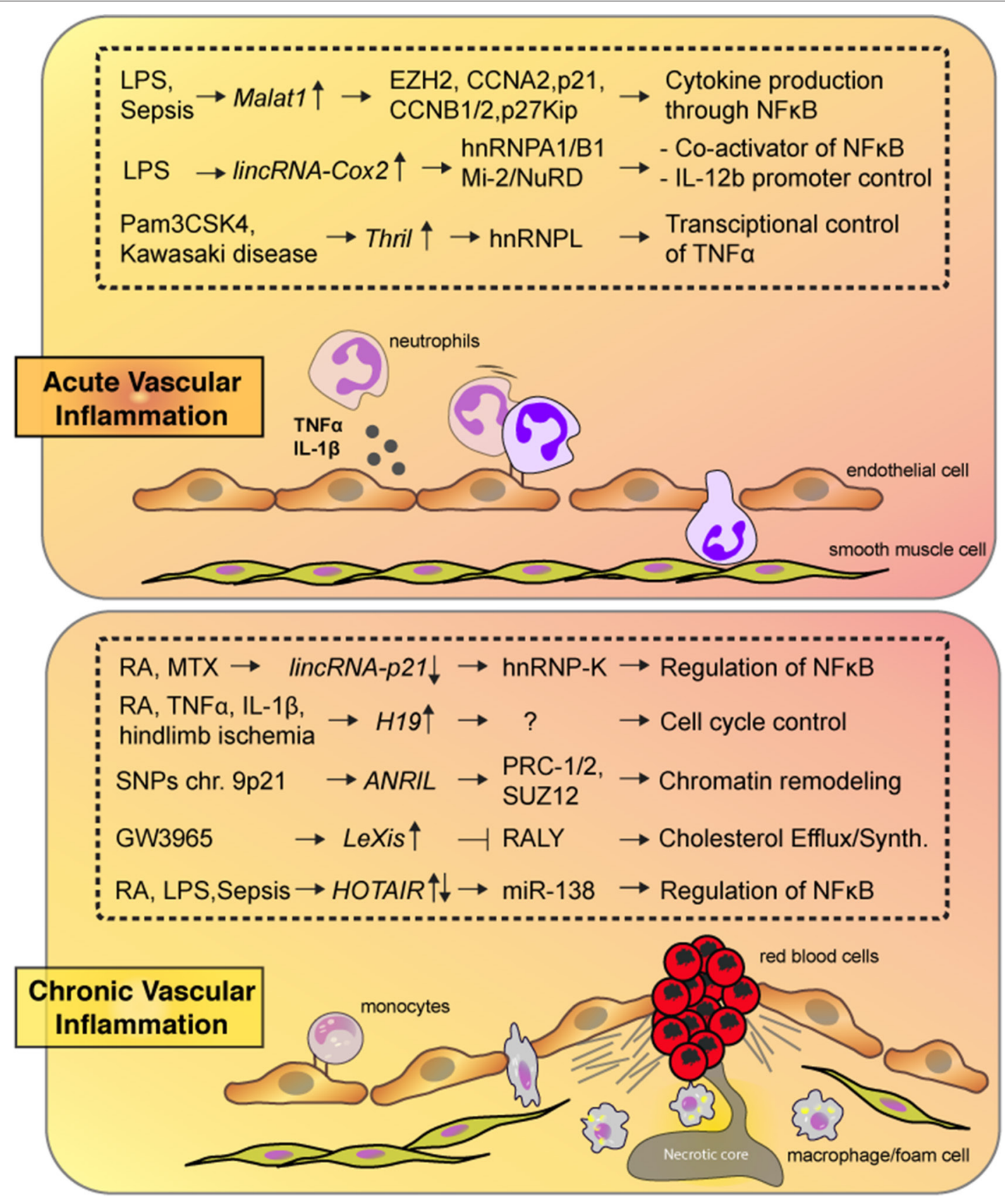

FIGURE 1 | LncRNAs in acute and chronic vascular inflammation. Highlighted IncRNAs from the text are shown along with their targets and biological consequences in response to acute (top) or chronic (bottom) vascular inflammation. 
increased in cardiomyocytes. HOTAIR silencing improved the cardiac function of septic mice, and markedly decreased TNFa production in the circulation and p65 phosphorylation in cardiomyocytes (59). These studies highlight a cell-type dependent role for HOTAIR in acute inflammation. Future studies will be required to identify potential compensatory mechanisms that may be activated in cardiovascular cell types versus chondrocytes after LPS activation.

\section{CONCLUSIONS AND FUTURE DIRECTIONS}

Despite current prevention interventions and guideline-based therapeutics, recurrent cardiovascular events after acute coronary syndromes remain elevated at $\sim 10 \%$ of patients within one year and $\sim 20 \%$ of patients within 36 months of initial presentation $(60,61)$. Biomarkers for systemic inflammation such as high sensitivity C-reactive protein (hsCRP) has been associated with increased risk for cardiovascular events (62). Although the role of inflammation in atherosclerosis has been identified over 150 years ago by Virchow (63), only recently has the "inflammation hypothesis" in atherosclerosis been specifically tested with an anti-inflammatory drug targeting IL- $1 \beta$. In the canakinumab anti-inflammatory thrombosis outcomes study (CANTOS), over 10,000 patients with elevated hsCRP at least 1 month post-myocardial infarction were randomized to receive the humanized monoclonal antibody canakinumab to neutralize IL-1 $\beta$ or placebo on top of usual therapy including statins. Impressively, recurrent cardiovascular events were reduced in the canakinumab treatment group independent of changes in lipid levels (64). As statin therapy lowers both LDL-C and inflammation (measured by hsCRP), CANTOS is the first clinical trial showing that lowering inflammation alone, without lowering LDL-C, significantly reduces cardiovascular events. Additional ongoing clinical trials using other anti-inflammatory drugs will likely provide further insights and impact clinical decision-making. For example, MTX is a widely used anti-inflammatory to treat RA patients (32). The cardiovascular inflammation reduction trial (CIRT) trial is currently investigating whether low dose of MTX administration will reduce the risk of cardiovascular events in patients with prior myocardial infraction and either type 2 diabetes or metabolic syndrome, all associated with chronic inflammation (ClinicalTrials.gov Identifier: NCT01594333) (65). Finally, the colchicine cardiovascular outcomes trial (COLCOT) will evaluate the long-term treatment of whether colchicine reduces rates of cardiovascular events in patients after myocardial infarction (ClinicalTrials.gov Identifier: NCT02551094).

Our understanding of the estimated 50,000 human lncRNAs in regulating acute and chronic inflammatory processes in the vasculature remains nascent, although accumulating studies demonstrate that lncRNAs hold great promise as important regulators of vascular inflammation. Apart from their expression profile, functional in vivo findings are key to understand their true translational value in acute and chronic inflammation of the vasculature and links with cardiovascular disease states (Figure 1). Furthermore, emerging technical advances provide the ability to uncover novel lncRNA interactors (12). ANRIL represents a SNP-associated loci, which may bear relevance for CVD and other diseases. $(39,66)$. Sensitive biomarkers have emerged for chronic inflammation burden such as CRP, SAA, and IL- 6 or vascular injury such as sICAM-1, sVCAM-1 and PTX3 (67). However, these two types of biomarkers tend to correlate weakly with each other. Because the expression of some lncRNAs track with stage-specific pathophysiological processes and they can be measured in serum (68), lncRNAs provide new avenues for diagnostics. For example, distinct lncRNAs may shed light on inflammatory subsets associated with systemic autoimmune diseases (e.g., RA, SLE) versus inflammation localized to the vessel wall (e.g., coronary or peripheral artery disease). Recent screening efforts of plasma from patients with CAD revealed a lncRNA named CoroMarker (AC100865.1) that was significantly increased in CAD patients compared to controls $(69,70)$. It will be of interest to examine whether this lncRNA is specific to CAD or increased in other chronic inflammatory diseases. While their translational value remains to be elucidated, ncRNA-based targeting strategies such as using antisense oligonucleotides (ASO) have already been approved for food and drug administration-approved drugs. Similar to miRNAs, lncRNAs are often differentially regulated in a cell-specific manner or in response to specific pathophysiological stimuli providing unique properties for therapeutic intervention. Challenges remain with the efficiency and specificity of delivery, which may be overcome by chemical modifications and/or nanoparticle carriers $(71,72)$. For example, ASOs that target liver-specific ligands [e.g., the liver-specific asialoglycoprotein receptor (ASGPR)] appear to confer strong efficacy and reasonable safety (73). Analogous paradigms for ASOs targeting vascular-specific ligands could provide novel therapeutics for vascular inflammation.

Collectively, because lncRNAs provide a new layer of control of protein-coding genes, IncRNAs may hold promise for uncovering novel pathophysiological insights, stage-specific biomarkers, and new targets for vascular inflammation in acute and chronic disease states.

\section{AUTHOR CONTRIBUTIONS}

$\mathrm{SH}$ and VS researched the data for the article, and significantly contributed to content and writing of the article. MF contributed to the conception of the article, and the writing, reviewing and editing of the draft manuscript.

\section{FUNDING}

This work was supported by the National Institutes of Health (HL115141, HL117994, HL134849, and GM115605 to MWF), the Arthur K. Watson Charitable Trust (to MWF), and the Dr. Ralph and Marian Falk Medical Research Trust (to MWF). 


\section{REFERENCES}

1. Libby P. Inflammation in atherosclerosis. Arterioscler Thromb Vasc Biol (2012) 32(9):2045-51. doi: 10.1161/ATVBAHA.108.179705

2. Hollan I, Meroni PL, Ahearn JM, Cohen Tervaert JW, Curran S, Goodyear CS, et al. Cardiovascular disease in autoimmune rheumatic diseases. Autoimmun Rev (2013) 12(10):1004-15. doi: 10.1016/j.autrev.2013.03.013

3. Yende S, Linde-Zwirble W, Mayr F, Weissfeld LA, Reis S, Angus DC. Risk of cardiovascular events in survivors of severe sepsis. Am J Respir Crit Care Med (2014) 189(9):1065-74. doi: 10.1164/rccm.201307-1321OC

4. Libby P, Ridker PM, Hansson GK. Progress and challenges in translating the biology of atherosclerosis. Nature (2011) 473(7347):317-25. doi: 10.1038/ nature 10146

5. Okazaki Y, Furuno M, Kasukawa T, Adachi J, Bono H, Kondo Set al. Analysis of the mouse transcriptome based on functional annotation of 60,770 full-length cDNAs. Nature (2002) 420(6915):563-73. doi: 10.1038/nature01266

6. Kapranov P, Cheng J, Dike S, Nix DA, Duttagupta R, Willingham AT, et al. RNA maps reveal new RNA classes and a possible function for pervasive transcription. Science (2007) 316(5830):1484-8. doi: 10.1126/science.1138341

7. Djebali S, Davis CA, Merkel A, Dobin A, Lassmann T, Mortazavi A, et al. Landscape of transcription in human cells. Nature (2012) 489(7414):101-8. doi: 10.1038 /nature 11233

8. Ulitsky I, Shkumatava A, Jan CH, Sive H, Bartel DP. Conserved function of lincRNAs in vertebrate embryonic development despite rapid sequence evolution. Cell (2011) 147(7):1537-50. doi: 10.1016/j.cell.2011.11.055

9. Washietl S, Kellis M, Garber M. Evolutionary dynamics and tissue specificity of human long noncoding RNAs in six mammals. Genome Res (2014) 24(4):616-28. doi: 10.1101 /gr.165035.113

10. Mercer TR, Gerhardt DJ, Dinger ME, Crawford J, Trapnell C, Jeddeloh JA, et al. Targeted RNA sequencing reveals the deep complexity of the human transcriptome. Nat Biotechnol (2011) 30(1):99-104. doi: 10.1038/nbt.2024

11. Wang K, Liu F, Zhou LY, Long B, Yuan SM, Wang Y, et al. The long noncoding RNA CHRF regulates cardiac hypertrophy by targeting miR-489. Circ Res (2014) 114(9):1377-88. doi: 10.1161/CIRCRESAHA.114.302476

12. Haemmig S, Feinberg MW. Targeting LncRNAs in cardiovascular disease: options and expeditions. Circ Res (2017) 120(4):620-3. doi: 10.1161/ CIRCRESAHA.116.310152

13. Haemmig S, Simion V, Yang D, Deng Y, Feinberg MW. Long noncoding RNAs in cardiovascular disease, diagnosis, and therapy. Curr Opin Cardiol (2017) 32(6):776-83. doi: 10.1097/HCO.0000000000000454

14. Muzio M, Polentarutti N, Bosisio D, Manoj Kumar PP, Mantovani A. Toll-like receptor family and signalling pathway. Biochem Soc Trans (2000) 28(5):563-6. doi: $10.1042 /$ bst0280563

15. Mullick AE, Soldau K, Kiosses WB, Bell TA, Tobias PS, Curtiss LK. Increased endothelial expression of Toll-like receptor 2 at sites of disturbed blood flow exacerbates early atherogenic events. J Exp Med (2008) 205(2):373-83. doi: 10.1084/jem.20071096

16. Keyel PA, Tkacheva OA, Larregina AT, Salter RD. Coordinate stimulation of macrophages by microparticles and TLR ligands induces foam cell formation. J Immunol (2012) 189(9):4621-9. doi: 10.4049/jimmunol.1200828

17. Zhao G, Su Z, Song D, Mao Y, Mao X. The long noncoding RNA MALAT1 regulates the lipopolysaccharide-induced inflammatory response through its interaction with NF-KB. FEBS Lett (2016) 590(17):2884-95. doi: 10.1002/18733468.12315

18. Yu Z, Rayile A, Zhang X, Li Y, Zhao Q. Ulinastatin protects against lipopolysaccharide-induced cardiac microvascular endothelial cell dysfunction via downregulation of lncRNA MALAT1 and EZH2 in sepsis. Int J Mol Med (2017) 39(5):1269-76. doi: 10.3892/ijmm.2017.2920

19. Zhuang YT, Xu DY, Wang GY, Sun JL, Huang Y, Wang SZ. IL-6 induced lncRNA MALAT1 enhances TNF- $\alpha$ expression in LPS-induced septic cardiomyocytes via activation of SAA3. Eur Rev Med Pharmacol Sci (2017) 21(2):302-9.

20. Puthanveetil P, Chen S, Feng B, Gautam A, Chakrabarti S. Long non-coding RNA MALAT1 regulates hyperglycaemia induced inflammatory process in the endothelial cells. J Cell Mol Med (2015) 19(6):1418-25. doi: 10.1111/ jcmm. 12576

21. Zhang B, Arun G, Mao YS, Lazar Z, Hung G, Bhattacharjee G, et al. The lncRNA Malat1 is dispensable for mouse development but its transcription plays a cis-regulatory role in the adult. Cell Rep (2012) 2(1):111-23. doi: 10.1016/j.celrep.2012.06.003

22. Carpenter S, Aiello D, Atianand MK, Ricci EP, Gandhi P, Hall LL, et al. A long noncoding RNA mediates both activation and repression of immune response genes. Science (2013) 341(6147):789-92. doi: 10.1126/science.1240925

23. Guttman M, Amit I, Garber M, French C, Lin MF, Feldser D, et al. Chromatin signature reveals over a thousand highly conserved large non-coding RNAs in mammals. Nature (2009) 458(7235):223-7. doi: 10.1038/nature07672

24. Covarrubias S, Robinson EK, Shapleigh B, Vollmers A, Katzman S, Hanley N. CRISPR/Cas9-based screening of long noncoding RNAs (lncRNAs) in macrophages with an NF-kappa B reporter. J Biol Chem (2017) 799155:jbc. M117.

25. Hu G, Gong AY, Wang Y, Ma S, Chen X, Chen J, et al. LincRNA-Cox2 promotes late inflammatory gene transcription in macrophages through modulating SWI/SNF-mediated chromatin remodeling. J Immunol (2016) 196(6):2799_ 808. doi: 10.4049/jimmunol.1502146

26. Tong Q, Gong AY, Zhang XT, Lin C, Ma S, Chen J, et al. LincRNA-Cox2 modulates TNF- $a$-induced transcription of Il12b gene in intestinal epithelial cells through regulation of $\mathrm{Mi}-2 / \mathrm{NuRD}$-mediated epigenetic histone modifications. Faseb J (2016) 30(3):1187-97. doi: 10.1096/f.15-279166

27. Li Z, Chao TC, Chang KY, Lin N, Patil VS, Shimizu C, et al. The long noncoding RNA THRIL regulates TNFa expression through its interaction with hnRNPL. Proc Natl Acad Sci USA (2014) 111(3):1002-7. doi: 10.1073/ pnas. 1313768111

28. Libby P. Mechanisms of acute coronary syndromes. N Engl J Med (2013) 369(9):883-4. doi: 10.1056/NEJMc1307806

29. Gimbrone MA, García-Cardeña G. Endothelial cell dysfunction and the pathobiology of atherosclerosis. Circ Res (2016) 118(4):620-36. doi: 10.1161/ CIRCRESAHA.115.306301

30. Mason JC, Libby P. Cardiovascular disease in patients with chronic inflammation: mechanisms underlying premature cardiovascular events in rheumatologic conditions. Eur Heart J (2015) 36(8):482-9. doi: 10.1093/ eurheartj/ehu403

31. Spurlock CF, Tossberg JT, Matlock BK, Olsen NJ, Aune TM. Methotrexate inhibits NF- $\mathrm{KB}$ activity via long intergenic (noncoding) RNA-p21 induction. Arthritis \& Rheumatology (2014) 66(11):2947-57. doi: 10.1002/art.38805

32. Segal R, Yaron M, Tartakovsky B. Methotrexate: mechanism of action in rheumatoid arthritis. Semin Arthritis Rheum (1990) 20(3):190-200. doi: 10.1016/0049-0172(90)90060-S

33. Huarte M, Guttman M, Feldser D, Garber M, Koziol MJ, Kenzelmann-Broz $\mathrm{D}$, et al. A large intergenic noncoding RNA induced by p53 mediates global gene repression in the p53 response. Cell (2010) 142(3):409-19. doi: 10.1016/j. cell.2010.06.040

34. Gottlieb TM, Jackson SP. The DNA-dependent protein kinase: requirement for DNA ends and association with Ku antigen. Cell (1993) 72(1):131-42. doi: 10.1016/0092-8674(93)90057-W

35. Maréchal A, Zou L. DNA damage sensing by the ATM and ATR kinases. Cold Spring Harb Perspect Biol (2013) 5(9):a012716. doi: 10.1101/cshperspect. a012716

36. Ju J, Naura AS, Errami Y, Zerfaoui M, Kim H, Kim JG, et al. Phosphorylation of $\mathrm{p} 50 \mathrm{NF}$-kappaB at a single serine residue by DNA-dependent protein kinase is critical for VCAM-1 expression upon TNF treatment. J Biol Chem (2010) 285(52):41152-60. doi: 10.1074/jbc.M110.158352

37. Stuhlmüller B, Kunisch E, Franz J, Martinez-Gamboa L, Hernandez MM, Pruss A, et al. Detection of oncofetal h19 RNA in rheumatoid arthritis synovial tissue. Am J Pathol (2003) 163(3):901-11. doi: 10.1016/S00029440(10)63450-5

38. Voellenkle C, Garcia-Manteiga JM, Pedrotti S, Perfetti A, de Toma I, da Silva $\mathrm{D}$, et al. Implication of Long noncoding RNAs in the endothelial cell response to hypoxia revealed by RNA-sequencing. Sci Rep (2016) 6:24141. doi: 10.1038/ srep 24141

39. Samani NJ, Erdmann J, Hall AS, Hengstenberg C, Mangino M, Mayer B, et al. Genomewide association analysis of coronary artery disease. $N$ Engl J Med (2007) 357(5):443-53. doi: 10.1056/NEJMoa072366

40. de Los Campos G, Gianola D, Allison DB. Predicting genetic predisposition in humans: the promise of whole-genome markers. Nat Rev Genet (2010) 11(12):880-6. doi: 10.1038/nrg2898 
41. Gschwendtner A, Bevan S, Cole JW, Plourde A, Matarin M, Ross-Adams H, et al. Sequence variants on chromosome 9p21.3 confer risk for atherosclerotic stroke. Ann Neurol (2009) 65(5):531-9. doi: 10.1002/ana.21590

42. Jarinova O, Stewart AF, Roberts R, Wells G, Lau P, Naing T, et al. Functional analysis of the chromosome 9p21.3 coronary artery disease risk locus. Arterioscler Thromb Vasc Biol (2009) 29(10):1671-7. doi: 10.1161/ ATVBAHA.109.189522

43. Helgadottir A, Thorleifsson G, Magnusson KP, Grétarsdottir S, Steinthorsdottir $\mathrm{V}$, Manolescu A, et al. The same sequence variant on 9p21 associates with myocardial infarction, abdominal aortic aneurysm and intracranial aneurysm. Nat Genet (2008) 40(2):217-24. doi: 10.1038/ng.72

44. Zeggini E, Weedon MN, Lindgren CM, Frayling TM, Elliott KS, Lango H, et al. Replication of genome-wide association signals in UK samples reveals risk loci for type 2 diabetes. Science (2007) 316(5829):1336-41. doi: 10.1126/ science. 1142364

45. Bishop DT, Demenais F, Iles MM, Harland M, Taylor JC, Corda E, et al. Genome-wide association study identifies three loci associated with melanoma risk. Nat Genet (2009) 41(8):920-5. doi: 10.1038/ng.411

46. Shete S, Hosking FJ, Robertson LB, Dobbins SE, Sanson M, Malmer B, et al. Genome-wide association study identifies five susceptibility loci for glioma. Nat Genet (2009) 41(8):899-904. doi: 10.1038/ng.407

47. Yu W, Gius D, Onyango P, Muldoon-Jacobs K, Karp J, Feinberg AP, et al. Epigenetic silencing of tumour suppressor gene p15 by its antisense RNA. Nature (2008) 451(7175):202-6. doi: 10.1038/nature06468

48. Holdt LM, Beutner F, Scholz M, Gielen S, Gäbel G, Bergert H, et al. ANRIL expression is associated with atherosclerosis risk at chromosome 9p21. Arterioscler Thromb Vasc Biol (2010) 30(3):620-7. doi: 10.1161/ ATVBAHA.109.196832

49. Congrains A, Kamide K, Oguro R, Yasuda O, Miyata K, Yamamoto E, et al. Genetic variants at the $9 \mathrm{p} 21$ locus contribute to atherosclerosis through modulation of ANRIL and CDKN2A/B. Atherosclerosis (2012) 220(2):449-55. doi: 10.1016/j.atherosclerosis.2011.11.017

50. Gil J, Peters G. Regulation of the INK4b-ARF-INK4a tumour suppressor locus: all for one or one for all. Nat Rev Mol Cell Biol (2006) 7(9):667-77. doi: 10.1038/ nrm1987

51. Yap KL, Li S, Muñoz-Cabello AM, Raguz S, Zeng L, Mujtaba S, et al. Molecular interplay of the noncoding RNA ANRIL and methylated histone $\mathrm{H} 3$ lysine 27 by polycomb CBX7 in transcriptional silencing of INK4a. Mol Cell (2010) 38(5):662-74. doi: 10.1016/j.molcel.2010.03.021

52. Burd CE, Jeck WR, Liu Y, Sanoff HK, Wang Z, Sharpless NE. Expression of linear and novel circular forms of an INK4/ARF-associated non-coding RNA correlates with atherosclerosis risk. PLoS Genet (2010) 6(12):e1001233. doi: 10.1371/journal.pgen.1001233

53. Holdt LM, Stahringer A, Sass K, Pichler G, Kulak NA, Wilfert W, et al. Circular non-coding RNA ANRIL modulates ribosomal RNA maturation and atherosclerosis in humans. Nat Commun (2016) 7:12429. doi: 10.1038/ ncomms12429

54. Hu YW, Zhao JY, Li SF, Huang JL, Qiu YR, Ma X, et al. RP5-833A20.1/miR382-5p/NFIA-dependent signal transduction pathway contributes to the regulation of cholesterol homeostasis and inflammatory reaction. Arterioscler Thromb Vasc Biol (2015) 35(1):87-101. doi: 10.1161/ATVBAHA.114.304296

55. Tontonoz P, Wu X, Jones M, Zhang Z, Salisbury D, Sallam T. Long noncoding RNA facilitated gene therapy reduces atherosclerosis in a Murine model of familial hypercholesterolemia. Circulation (2017) 136(8):776-8. doi: 10.1161/ CIRCULATIONAHA.117.029002

56. Sallam T, Jones MC, Gilliland T, Zhang L, Wu X, Eskin A, et al. Feedback modulation of cholesterol metabolism by the lipid-responsive non-coding RNA LeXis. Nature (2016) 534(7605):124-8. doi: 10.1038/nature17674

57. Song J, Kim D, Han J, Kim Y, Lee M, Jin EJ. PBMC and exosome-derived Hotair is a critical regulator and potent marker for rheumatoid arthritis. Clin Exp Med (2015) 15(1):121-6. doi: 10.1007/s10238-013-0271-4
58. Zhang HJ, Wei QF, Wang SJ, Zhang HJ, Zhang XY, Geng Q, et al. LncRNA HOTAIR alleviates rheumatoid arthritis by targeting miR-138 and inactivating NF- $\kappa$ B pathway. Int Immunopharmacol (2017) 50:283-90. doi: 10.1016/j. intimp.2017.06.021

59. Wu H, Liu J, Li W, Liu G, Li Z. LncRNA-HOTAIR promotes TNF- $\alpha$ production in cardiomyocytes of LPS-induced sepsis mice by activating NF- $\kappa \mathrm{B}$ pathway. Biochem Biophys Res Commun (2016) 471(1):240-6. doi: 10.1016/j. bbrc.2016.01.117

60. Wallentin L, Becker RC, Budaj A, Cannon CP, Emanuelsson H, Held C, et al. Ticagrelor versus clopidogrel in patients with acute coronary syndromes. $\mathrm{N}$ Engl J Med (2009) 361(11):1045-57. doi: 10.1056/NEJMoa0904327

61. Jernberg T, Hasvold P, Henriksson M, Hjelm H, Thuresson M, Janzon M. Cardiovascular risk in post-myocardial infarction patients: nationwide real world data demonstrate the importance of a long-term perspective. Eur Heart $J$ (2015) 36(19):1163-70. doi: 10.1093/eurheartj/ehu505

62. Ridker PM, Hennekens CH, Buring JE, Rifai N. C-reactive protein and other markers of inflammation in the prediction of cardiovascular disease in women. N Engl J Med (2000) 342(12):836-43. doi: 10.1056/NEJM200003233421202

63. Virchow RLK. Cellular pathology as based upon physiological and pathological histology. New York: Dover Publications (1971).

64. Ridker PM, Everett BM, Thuren T, MacFadyen JG, Chang WH, Ballantyne C, et al. Antiinflammatory therapy with canakinumab for atherosclerotic disease. N Engl J Med (2017) 377(12):1119-31. doi: 10.1056/NEJMoa1707914

65. Everett BM, Pradhan AD, Solomon DH, Paynter N, Macfadyen J, Zaharris E, et al. Rationale and design of the Cardiovascular Inflammation Reduction Trial: a test of the inflammatory hypothesis of atherothrombosis. Am. Heart J. (2013) 166(2):199-207.e15. doi: 10.1016/j.ahj.2013.03.018

66. Ward LD, Kellis M. HaploReg: a resource for exploring chromatin states, conservation, and regulatory motif alterations within sets of genetically linked variants. Nucleic Acids Res (2012) 40(Database issue):D930-34. doi: 10.1093/ nar/gkr917

67. Yasunaga T, Ikeda S, Koga S, Nakata T, Yoshida T, Masuda N, et al. Plasma pentraxin 3 is a more potent predictor of endothelial dysfunction than highsensitive C-reactive protein. Int Heart J (2014) 55(2):160-4. doi: 10.1536/ ihj.13-253

68. Viereck J, Kumarswamy R, Foinquinos A, Xiao K, Avramopoulos P, Kunz M, et al. Long noncoding RNA Chast promotes cardiac remodeling. Sci Transl Med (2016) 8(326):ra22. doi: 10.1126/scitranslmed.aaf1475

69. Yang Y, Cai Y, Wu G, Chen X, Liu Y, Wang X, et al. Plasma long non-coding RNA, CoroMarker, a novel biomarker for diagnosis of coronary artery disease. Clin Sci (2015) 129(8):675-85. doi: 10.1042/CS20150121

70. Cai Y, Yang Y, Chen X, Wu G, Zhang X, Liu Y, et al. Circulating 'IncRNA OTTHUMT00000387022' from monocytes as a novel biomarker for coronary artery disease. Cardiovasc Res (2016) 112(3):714-24. doi: 10.1093/cvr/cvw022

71. Aagaard L, Rossi JJ. RNAi therapeutics: principles, prospects and challenges. Adv Drug Deliv Rev (2007) 59(2-3):75-86. doi: 10.1016/j.addr.2007.03.005

72. Chery J. RNA therapeutics: RNAi and antisense mechanisms and clinical applications. Postdoc J (2016) 4(7):35-50. doi: 10.14304/SURYA.JPR.V4N7.5

73. Feinberg MW. No small task: therapeutic targeting of $\mathrm{Lp}(\mathrm{a})$ for cardiovascular disease. Lancet (2016) 388(10057):2211-2. doi: 10.1016/S01406736(16)31329-0

Conflict of Interest Statement: The authors declare that the research was conducted in the absence of any commercial or financial relationships that could be construed as a potential conflict of interest.

Copyright (c) 2018 Haemmig, Simion and Feinberg. This is an open-access article distributed under the terms of the Creative Commons Attribution License (CC BY). The use, distribution or reproduction in other forums is permitted, provided the original author (s) and the copyright owner are credited and that the original publication in this journal is cited, in accordance with accepted academic practice. No use, distribution or reproduction is permitted which does not comply with these terms. 